\title{
ATMOSPHERIC GAS ABSORPTION KNOWLEDGE IN THE SUBMILLIMETER Modeling, Field Measurements, and Uncertainty Quantification
}

\author{
Vinia Mattioli, Christophe Accadia, Catherine Prigent, Susanne Crewell, Alan Geer, Patrick Eriksson, \\ Stuart Fox, Juan R. Pardo, Eli J. Mlawer, Maria Cadeddu, Michael Bremer, Carlos De Breuck, \\ Alain Smette, Domenico Cimini, Emma Turner, Mario Mech, Frank S. Marzano, Pascal Brunel, \\ Jerome Vidot, Ralf Bennartz, Tobias Wehr, Sabatino Di Michele, and Viju O. John
}

T he first of the European Organisation for the Exploitation of Meteorological Satellites (EUMETSAT) Polar System (EPS) Second Generation (EPS-SG) meteorological satellites will be launched in the 2023 timeframe and will include the Ice Cloud Imager (ICI), a passive conical-scanning radiometer observing in the microwave to submillimeter wave range of the spectrum with 11 channels from 183 to $664 \mathrm{GHz}$. It will be the first operational meteorological instrument with frequencies in the submillimeter range, which is between the microwave and infrared spectrums. These frequencies are sensitive to atmospheric temperature, moisture, and hydrometeors, with unique information on cloud ice. The primary objective of the ICI is the quantification of cloud ice in support of climate monitoring, the validation of ice cloud models, and the parameterization of ice clouds in weather and climate models.

To make quantitative use of observations provided by this innovative sensor requires a thorough understanding of atmospheric gas absorption and emission, dominated at these frequencies by oxygen and water vapor. Figure 1 shows downward-looking clear-sky brightness temperature calculations at the ICI viewing angle. Absorption models representing the absorption and emission of electromagnetic radiation by atmospheric gas constituents must be

\section{GAS ABSORPTION KNOWLEDGE IN THE} SUBMILLIMETER REGION

WHAT: Members of the atmospheric and astronomical science communities met to review the current state of the art of the submillimeter spectral region. Knowledge of gas spectroscopy is still questionable at these frequencies but is important to fully exploit upcoming meteorological satellite measurements.

WHEN: 6-7 December 2018

WHERE: Darmstadt, Germany

properly characterized in order to simulate the clear-sky background within which cloud-induced scattering signals will be detected. An error in the absorption model will impact ICI's ability to detect thin ice clouds, and will also affect the quality of information obtained on humidity. However, to date, there has been little validation of atmospheric absorption models in the submillimeter wave range (above $300 \mathrm{GHz}$ ), and even at high microwave frequencies like $183 \mathrm{GHz}$ there are questions on the quality of the current spectroscopy knowledge.

EUMETSAT organized a workshop on the state of atmospheric gas absorption modeling, with 24 


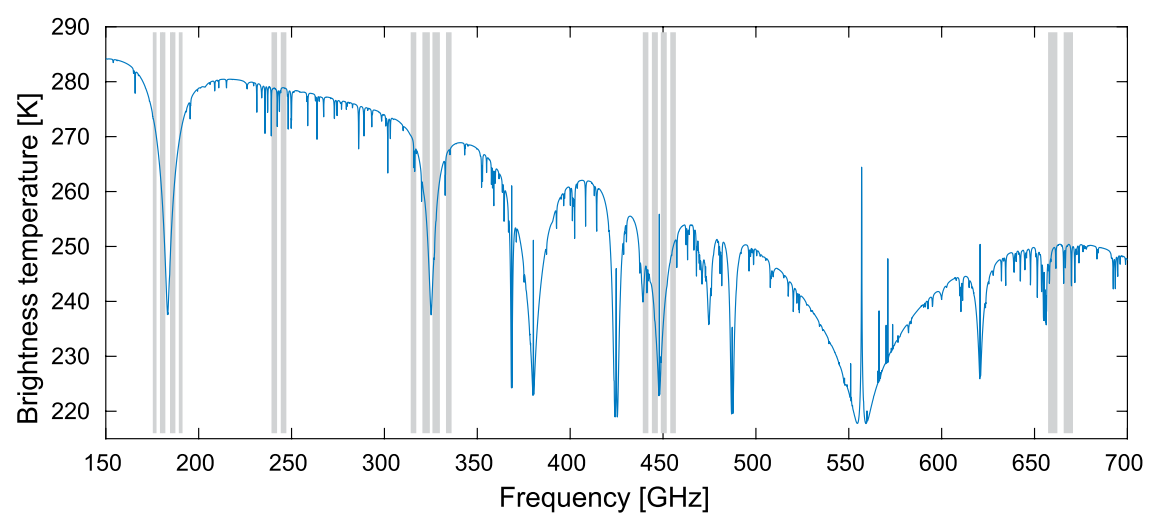

FIG. I. Brightness temperature simulations calculated at the $\mathrm{ICl}$ viewing angle for a midlatitude summer scenario in the frequency range covered by ICI. ICI double sideband channels are highlighted as gray bars. (Based on data from HITRAN, calculated by the ARTS software.)

attendees from the atmospheric and astronomical science communities, bringing together spectroscopists, experimentalists, radiative transfer modelers, and operational meteorologists. The aim of the workshop was to review available field measurements, key absorption models, their strengths and limitations, and methods to evaluate these models with the ultimate goal of narrowing down their uncertainties in the microwave to submillimeter range.

FIELD MEASUREMENTS. Field measurements include up-looking (ground-based and aircraft) and down-looking (from aircraft) perspectives and are available within both atmospheric and astronomical communities. Due to the strong water vapor absorption at submillimeter wavelengths, ground-based measurements are limited to very dry conditions. Therefore, submillimeter telescopes for astronomy are situated or planned at altitudes above the wet scale height of $\sim 2 \mathrm{~km}$, preferentially in dry climates: Chajnantor, Chile (ALMA, APEX), Mauna Kea, Hawaii (SMA, JCMT), Pico Veleta, Spain (IRAM-30M), Plateau de Bure, France (NOEMA), Summit Station, Greenland (GLT), and South Pole Station, Antarctica (SPT). The U.S. Department of Energy (DoE) Atmospheric Radiation Measurement (ARM) Program's (www .arm.gov/) ground-based radiometers have decadal time series of observations between 22 and $183 \mathrm{GHz}$ and have been used to validate several parameters and coefficients of absorption models, including $\mathrm{H}_{2} \mathrm{O}$ line parameters, water vapor continuum, $\mathrm{O}_{2}$ line coupling coefficients, and supercooled liquid water by simultaneous measurements of the atmospheric state and brightness temperatures. The international Arctic drift expedition MOSAIC (www.mosaic-expedition .org/) planned for 2019/20 will provide high-quality atmospheric profiles together with microwave measurements up to $340 \mathrm{GHz}$.

For astronomy, models are required to correct for atmospheric transmission and phase delays at
AfFiliations: MatTIOLI, ACCADIA, DI MiChele, AND JOHN-European Organisation for the Exploitation of Meteorological Satellites, Darmstadt, Germany; PRIGENT-Département de Radioastronomie Millimétrique, Observatoire de Paris, Paris, France; CREWELL AND $\mathrm{MECH}$ - Institute for Geophysics and Meteorology, University of Cologne, Cologne, Germany; GeER-European Centre for MediumRange Weather Forecasts, Reading, United Kingdom; ERIKSSONDepartment of Space, Earth and Environment, Chalmers University of Technology, Gothenburg, Sweden; FOX AND TURner—Met Office, Exeter, United Kingdom; PARDO_Instituto de Física Fundamental, Consejo Superior de Investigaciones Cientificas, Madrid, Spain; MLAWER-Atmospheric and Environmental Research, Lexington, Massachusetts; CADEDDU-Environmental Science Division, Argonne National Laboratory, Argonne, Illinois; BrEMER-Institut de Radio Astronomie Millimétrique, Saint Martin d'Hères, France; De BREUCK-European Southern Observatory, Garching bei München, Germany; SMETTE-European Southern Observatory, Santiago, Chile; CIMINI-National Research Council of Italy, Institute of Methodologies for Environmental Analysis, Potenza, and Center of Excellence CETEMPS, University of L'Aquila, L'Aquila, Italy; MARZANO-Department of Information Engineering, Sapienza University of Rome, Rome, and Center of Excellence CETEMPS, University of L'Aquila, L'Aquila, Italy; BRUNEL AND VIDOT-Centre National de Recherches Météorologiques, Université de Toulouse, Météo-France, CNRS, Lannion, France; BenNARTZ-Earth and Environmental Sciences, Vanderbilt University, Nashville, Tennessee, and Space Science and Engineering Center, University of WisconsinMadison, Madison, Wisconsin; WeHR-European Space Research and Technology Centre, European Space Agency, Noordwijk, Netherlands

CORRESPONDING AUTHOR: Vinia Mattioli, vinia.mattioli@eumetsat.int

DOI:10.II75/BAMS-D-19-0074.I

In final form 24 January 2019

(C)2019 American Meteorological Society

For information regarding reuse of this content and general copyright information, consult the AMS Copyright Policy. 
submillimeter wavelengths. The astronomical community has extensive ground-based observation datasets from submillimeter telescopes and other instruments (http://archive.eso.org/wdb/wdb/asm/meteo_apex /form) used to infer the atmospheric profile, making use of both Fourier transform spectrometers and broadband radiometers well into the Terahertz range. These could be very useful to refine and evaluate absorption models.

Aircraft down-looking measurements are available among others from the International Submillimetre Airborne Radiometer (ISMAR) on board the U.K. Facility for Airborne Atmospheric Measurements (FAAM) aircraft with channels between 118 and $664 \mathrm{GHZ}$, and up to $874 \mathrm{GHz}$ in the future (Fox et al. 2017). Aircraft flights can replicate the satellite view, are flexible in altitude, and can provide in situ and dropsonde data. However, for down-looking views the uncertainty in the surface contribution and the relatively small temperature contrast between the surface and atmosphere reduces the sensitivity to the atmospheric absorption models. The up-looking approach has the advantage of avoiding surface contributions and the background brightness temperature is very low, thus providing higher sensitivity to atmospheric absorption models.

LACK OF A REFERENCE MODEL. Radiative transfer models (RTMs) implement the radiative transfer equation to compute simulated brightness temperatures, resorting to atmospheric absorption models to determine the contribution of the atmospheric gases. RTMs are used for the retrieval of atmospheric parameters, the validation of instrument measurements, and their assimilation in NWP models.

Line-by-line RTMs contain absorption models that numerically integrate individual absorption lines. These include the knowledge of resonant line strengths from quantum mechanics, line profiles, and line widths from spectroscopic laboratory measurements, and a nonresonant continuous absorption usually known as "continuum," which is empirically derived from fits to measurements. The way the continuum is modeled depends upon line choices, and line shape frequency cutoff. Given profiles of temperature, moisture, and other absorbers (most relevantly oxygen and ozone) line-by-line models first compute the atmospheric transmittance for each layer. From these, the up- and down-looking radiances and brightness temperatures are computed, as well as the full atmospheric transmittance at any given frequency.
Conversely, fast RTMs use parameterized transmittance, trained with statistical fit against lineby-line transmittance for diverse atmospheres and for specific instrument characteristics. NWP applications require fast parameterized RTMs, and their evaluation is a prerequisite before operational deployment.

Underlying most of the RTMs used by the atmospheric science community is a combination of the high-resolution transmission molecular absorption (HITRAN) line database and lines as in Atmospheric and Environmental Research's MonoRTM (Clough et al. 2005), Liebe's Millimeter-Wave Propagation Model (MPM), and Rosenkranz's absorption model (Rosenkranz 2017). Widely adopted by astronomers as reference is the Atmospheric Transmission at Microwaves (ATM) model (Pardo et al. 2001), which has its own quantum-mechanical calculations and experimental continuum based on Fourier Transform Spectroscopy (FTS) experiments.

However, due to continuous developments in laboratory spectroscopy and the uncertainty associated with individual model parameters, no consensus on a "reference" model in the submillimeter range exists yet.

AMSUTRAN (Turner et al. 2019) was a critical part of our survey because it is the line-by-line model used for the training of the fast parameterized radiative transfer model Radiative Transfer for TOVS (RTTOV; Saunders et al. 2018), which will be used in the operational exploitation of ICI data. AMSUTRAN is currently undergoing significant redevelopment with a focus above $200 \mathrm{GHz}$. This potentially includes the addition of more lines including ozone, updated line parameters, and a new water vapor continuum parameterization. Line-by-line RTMs such as Atmospheric Radiative Transfer Simulator (ARTS; Buehler et al. 2018) and Passive and Active Microwave Transfer model (PAMTRA; http://github .com/igmk/pamtra/) offer different selections of absorption models with line parameters and continuum parameterizations, which is required for characterization of modeling uncertainties and for flexibility when performing comparisons between simulation tools. An intercomparison of different options for line and continuum parameters showed encouraging results for down-looking satellite simulations, with differences lower than $0.5 \mathrm{~K}$ for the absorption lines and within -0.5 to $+1.5 \mathrm{~K}$ for different continuum candidates. Omitting ozone lines could lead to errors of up to tens of kelvin in brightness temperature. For ICI specifically, the error is up to $1.5 \mathrm{~K}$ in the 664 $\mathrm{GHz}$ channels. 
Evidence of ongoing uncertainties was seen in Atmospheric and Environmental Research's development of the MT_CKD continuum model, which is not observationally constrained between 183 and $600 \mathrm{GHz}$. Here the latest validation results, made at higher water vapor amounts than before, are challenging earlier confidence in the model.

QUANTIFYING UNCERTAINTY. Uncertainty in our spectroscopic knowledge may be investigated through several different approaches. One route is comparing simulations against reference measurements (such as airborne ISMAR or astronomical measurements). Up-looking observations (either from ground or aircraft) are preferable, as they are nearly unaffected by the surface emission and reflection, and thus are more sensitive than down-looking to spectroscopy differences. However, this approach depends critically on the quality of the atmospheric profile data that are used as input to the calculation. For example, measurement uncertainty in radiosonde water vapor profiles is of the same magnitude as the spectroscopic uncertainty we want to resolve. One proposal to mitigate this issue was to investigate the consistency of $1 \mathrm{D}$-Var retrievals from the radiances themselves.

Along similar lines, NWP centers directly compare modeled and observed radiances producing the observation minus background $(O-B)$ statistics that are part of the data assimilation process. $O-B$ statistics have been used to detect instrument calibration issues and to identify possible spectroscopic errors, but it can be hard to separate systematic errors in the forecast model and observational data that go into the data assimilation process.

An alternative approach is to propagate the uncertainties of the spectroscopic parameters within absorption models into RTM computations. Rigorously, not only the uncertainty but the full covariance among the spectroscopic parameters is needed, since uncertainties in spectroscopic parameters can be strongly (positively and negatively) correlated. Such a method has been applied to ground-based simulations at $20-60 \mathrm{GHz}$ (Cimini et al. 2018) and the initial extension to down-looking millimeter-wave range was proposed at the workshop.

CONCLUSIONS AND RECOMMENDATION. At present there is no consensus over a reference atmospheric absorption model. Further, the choice of absorption model is not always separable from the choice of RTM. During the workshop, the following RTMs were selected for further investigation, and they encapsulate a range of absorption models. The Atmospheric and Environmental Research MonoRTM is widely used and provided good results in the analysis for the AMSUTRAN development and when compared with ISMAR data. Still, recent developments showed during the workshop are currently challenging Atmospheric and Environmental Research's previous confidence. Rosenkranz's (2017) recent update has been used in the uncertainty propagation analysis and in the development of the ground-based version of RTTOV. ATM is widely tested by the astronomers and is also offered for comparison. Further, the choice of atmospheric constituents included in the model is important: apart from oxygen and water vapor, ozone lines have a nonnegligible impact, and need to be included.

New laboratory measurements aiming at refining the values and uncertainties of spectroscopy parameters are highly desirable. It is recommended that spectroscopists provide full uncertainty on the parameters including possible correlations with other relevant parameters.

It was proposed to collate a carefully selected database of different observations for further intercomparison studies of those models. There is a need for consistency: across frequencies, up- and downlooking, ground-based (including data from radio telescopes), and space-based observations.

Also, it was noted that radiosonde uncertainty is now carefully quantified within the Global Climate Observing System (GCOS) Reference Upper-Air Network (GRUAN) efforts. It might therefore be worth repeating or reprocessing data from previous ground-based field campaigns at GRUAN sites.

Additionally, it could also be valuable validating simulations against limb sounding submillimeter observations, as provided by the Submillimeter Radiometer aboard the Odin mission (http://odin.rss .chalmers.se/) or the Aura Microwave Limb Sounder (https://mls.jpl.nasa.gov/). Comparison against limb sounding submillimeter observations data has not yet been performed.

The agreement on a reference absorption model within the community was identified as the highest priority. Once this is available the methodology to derive a fast model can be optimized.

ACKNOWLEDGMENTS. The authors thank Sreerekha Thonipparambil for verifying the contents of this meeting summary. The workshop was hosted by the European Organisation for the Exploitation of Meteorological Satellites (EUMETSAT), Darmstadt, Germany. Maria Cadeddu is supported by the U.S. Department of Energy, Office of 
Science, Office of Biological and Environmental Research, Atmospheric Radiation Measurement Infrastructure Basic Energy Sciences, under contract DE-AC02-06CH11357. Patrick Ericksson is was supported by the Swedish National Space Agency. Ralf Bennartz was supported by NASA USPI Grant NNX17AJ09G.

\section{REFERENCES}

Buehler, S. A., J. Mendrok, P. Eriksson, A. Perrin, R. Larsson, and O. Lemke, 2018: ARTS, the Atmospheric Radiative Transfer Simulator - version 2.2, the planetary toolbox edition. Geosci. Model Dev., 11, 1537-1556, https://doi.org/10.5194/gmd-11-1537 -2018 .

Cimini, D., P. W. Rosenkranz, M. Y. Tretyakov, M. A. Koshelev, and F. Romano, 2018: Uncertainty of atmospheric microwave absorption model: Impact on ground-based radiometer simulations and retrievals. Atmos. Chem. Phys., 18, 15231-15259, https://doi .org/10.5194/acp-18-15231-2018.

Clough, S. A., M. W. Shephard, E. J. Mlawer, J. S. Delamere, M. J. Iacono, K. Cady-Pereira, S. Boukabara, and P. D. Brown, 2005: Atmospheric radiative transfer modeling: A summary of the AER codes. J. Quant. Spectrosc. Radiat. Transf., 91, 233-244, https://doi.org/10.1016/j.jqsrt.2004.05 .058 .

Fox, S., and Coauthors, 2017: ISMAR: An airborne submillimetre radiometer. Atmos. Meas. Tech., 10 , 477-490, https://doi.org/10.5194/amt-10-477-2017.

Pardo, J. R., J. Cernicharo, and E. Serabyn, 2001: Atmospheric transmission at microwaves (ATM): An improved model for millimeter/submillimeter applications. IEEE Trans. Antennas Propag., 49, 1683-1694, https://doi.org/10.1109/8.982447.

Rosenkranz, P. W., 2017: Line-by-line microwave radiative transfer (non-scattering). Remote Sensing Code Library, https://doi.org/10.21982/M81013.

Saunders, R., and Coauthors, 2018: An update on the RTTOV fast radiative transfer model (currently at version 12). Geosci. Model Dev., 11, 2717-2737, https:// doi.org/10.5194/gmd-11-2717-2018.

Turner, E. C., P. Rayer, and R. Saunders, 2019: AMSUTRAN: A microwave transmittance code for satellite remote sensing. J. Quant. Spectrosc. Radiat. Transf., 227, 117-129, https://doi.org/10.1016/j .jqsrt.2019.02.013. 